\title{
Integrating a Tracking Camera in the OR Using the IEEE 11073-SDC Communication Standard
}

\author{
Manuel Vossel $\mathrm{MSc}^{1}$, Matías de la Fuente $\mathrm{PhD}^{1}$, \\ Dario Wieschebrock BSc ${ }^{1}$, Okan Yilmaz BSc ${ }^{1}$, \\ Klaus Radermacher $\mathrm{PhD}^{1}$, and Armin $\mathrm{Jan} ß \mathrm{PhD}^{1}$ \\ ${ }^{1}$ Chair of Medical Engineering, Helmholtz Institute for Biomedical Engineering, \\ RWTH Aachen University, Germany \\ vosselehia.rwth-aachen. de
}

\begin{abstract}
With the increasing spread of computer assisted surgery, more and more modern operating rooms are equipped with navigation systems, each coming with its own tracking camera. Since those cameras are part of the closed monolithic navigation system, they can't be used for other applications than the one intended by the supplier. With the novel service oriented device connectivity standard (IEEE 11073-SDC), introduced by the OR.NET initiative (www.ornet.org), needless double procurements could be avoided and multiple systems could use the same camera that - similar to OR lights - could be installed as a standard equipment in each OR. This would decrease the cost-to-benefit ratio also of new applications that would currently as such not justify to acquire a proprietary tracking camera.

While the integration of a tracking camera to an open medical device IT network can open up for new applications, it should on the other hand not impair the usability and the safety of the navigation system. Therefore, a low latency must be guaranteed between tracking camera and navigational display.

This paper evaluates the integration of an atracsys fusionTrack 500 tracking camera into the OR.NETwork. The response time from a change in the real world to the reception of the corresponding data package is measured to determine the feasibility of an integration without impairing current navigational tasks.

The results show that, as long as the underlying network infrastructure is not at its capacity limit, latencies below $60 \mathrm{~ms}$ are achieved. Therefore, the integration of a tracking camera for navigational tasks is feasible.
\end{abstract}




\section{Introduction}

With the increasing spread of computer assisted surgery, more and more modern operating rooms are equipped with navigation systems, each coming with its own tracking camera. Since those cameras are part of the closed monolithic navigation system, they can't be used for other applications than the one intended by the supplier. Thus, if one operating room is for example used for total hip arthroplasty with the orthopedic navigation system of company A as well as for spinal fusion with the neurosurgical navigation system of company $\mathrm{B}$, both using basically identical tracking cameras of company $\mathrm{N}$, investments (10-15k $€$ each) and handling for two almost identical tracking systems in the OR are necessary and nevertheless none of these tracking systems can be used for other applications. Following the OR.NET interoperability approach (Golatowski 2018; Mildner 2015; Vitting 2018; Dell'Anna 2016; www.ornet.org), this waste of resources could be avoided and one single tracking camera could be used by different systems and applications. This issue had been addressed by Ibach (Ibach 2011; Ibach 2009), who partly integrated a tracking system into a service oriented architecture (SOA). A dedicated server works as a tracking service provider, having one or more tracking cameras attached. Each camera can be configured within the open network by one consumer, i.e. the navigation system, and can send out the tracking data using direct TCP/IP or UDP packages. With this approach, each tracking camera is exclusively controlled by one consumer, i.e. navigation system, at a time.

However, central tracking services could be useful for many other applications in the OR. New applications become possible that are currently not worth acquiring a proprietary tracking camera due to a high cost-to-benefit ratio. With just an app on an OR.NET-ready surgical desktop or tablet workstation and a standard pointer, a surgeon will be able to measure anatomical dimensions like the length of a patient's limb, the length of an incision or even the rough area of a burn. Also new concepts for user interactions with devices independent from a specific navigation system are possible. Examples such as the Remote Pointer (Janß 2009) or the Zero-dose C-arm navigation application (Fuente 2007; Ladenburger 2012) are only some among many others. Since those examples require the tracking system to be configured by multiple consumers simultaneously, the feasibility of a full integration into the service oriented IEEE 11073-SDC communication standard family (IEEE 11073-10207, -20701, -20702) developed in the context of the OR.NET initiative (www.ornet.org) is investigated in this paper. This is in contrast to the approach proposed by Ibach, where only one consumer had the exclusive right to control the tracking camera and change parameters like the geometry of the tracked arrays.

While the integration of a tracking camera to an open medical device IT network can open up for new applications, it should on the other hand not impair the usability and the safety of e.g. a navigation system. Regarding the usability, it is widely accepted that a latency in the visual feedback impairs the outcome of the task to be performed (Luck 2006; Chen 2007). Therefore, a low latency must be guaranteed. MacKenzie and Ware (MacKenzie 1993) performed a study where subjects had to move the computer cursor towards a target, with different latencies ranging from $8.3 \mathrm{~ms}$ to $225 \mathrm{~ms}$, measuring task completion time and error rate. Already with a latency of $75 \mathrm{~ms}$ an increase in error rate and completion time could be measured.

In contrast to manual navigational tasks, also robotic applications using an OR.NET integrated tracking camera are imaginable (Vossel 2017). At the Chair of Medical Engineering, RWTH Aachen University, robotic systems are being developed with the tracking camera being an integral part of the control loop. Real time control for smooth movement of the end-effector is implemented on a dSpace system (dSpace, Paderborn, Germany) with latencies of the tracking signal between $6 \mathrm{~ms}$ and $8 \mathrm{~ms}$.

Regarding the safety of integrating a tracking camera into an open medical device IT network, the development of tools and methods for the testing and approval of components and systems for the operating room of the future is a major objective of the ongoing work of the OR.NET initiative.

This paper evaluates the integration of a fusionTrack 500 tracking camera (Atracsys LLC, Puidoux, $\mathrm{CH}$ ) into the OR.NETwork. The response time from a change in the real world to the reception of the 
corresponding data package is measured to determine the feasibility of an integration without impairing current navigational tasks.

\section{Material and Methods}

To evaluate the latency of an OR.NET based tracking system, a setup has been selected with two real time Linux systems as consumer and provider, an atracsys fusionTrack500 tracking camera, and a single infrared (IR) LED, used as a single fiducial. The consumer and provider software was implemented using the SDCLib/C by SurgiTAIX AG, Herzogenrath, Germany (Besting 2018; www.surgitaix.com). Details of the underlying service oriented architecture are described in (Ibach 2008; Kasparick 2015). The IR LED can be controlled by the consumer Linux system. The tracking camera is connected to the provider Linux system, which sends out the number of all stray fiducials in the working volume of the camera. This metric is published in the OR.NETwork by the provider with a $60 \mathrm{~Hz}$ update rate.

After the IR LED is turned on by the consumer, it gets recognized by the tracking camera as a fiducial and this information is sent over the OR.NETwork. The consumer measures the time between turning on the LED and receiving the respective data through the OR.NETwork.

This latency test is carried out in four different scenarios. In the first scenario, only the tracking consumer and the tracking service provider are connected to the OR.NETwork. For the second scenario, ten medical devices (OR table, footswitch, HF unit, shaver, video endoscope, endoscope light, insufflator, endoscope documentation unit, OR lamp and OR camera) as well as a central surgical workstation are connected to the network. The workstation makes a discovery scan every 30 seconds that is confirmed by each connected device in the network - i.e. the more connected devices there are, the higher is the load on the network. Apart from the traffic generated by the discovery messages and the tracking camera, no other apparent traffic is generated on the network in the second scenario. During the third scenario, the parameters of the connected medical devices are changed multiple times during the latency test and the shaver is operated by the footswitch. Additionally, one provider of a simulated device was implemented pushing out 200 values every second, imitating for example an anaesthetic machine periodically sending vital parameters. Five additional generic providers are activated, sending out 5 values every $200 \mathrm{~ms}$ each. In the fourth scenario, the capacity limit of the OR.NETwork is tested. Therefore, 25 additional generic providers, each sending out 5 values every $200 \mathrm{~ms}$, are added, resulting in a total of 31 generic providers.

As a reference, another setup is tested where the tracking camera is directly connected to the real time Linux system controlling the IR LED. This setup measures the bare latency of the tracking camera and the software reading out its data.

In each test scenario, 1,000 latency measurements are taken. Mean latency, standard deviation, minimum, maximum and $95 \%$ maximum latency are calculated for these test samples. 


\section{Results}

The results of the latency tests are summarized in Table 1.

\begin{tabular}{l|lrrrr}
$\begin{array}{l}\text { Test Scenario / Connected } \\
\text { Devices to the OR.NETwork }\end{array}$ & $\begin{array}{l}\text { Mean } \\
\text { latency }\end{array}$ & $\begin{array}{l}\text { Standard } \\
\text { deviation }\end{array}$ & $\begin{array}{l}\text { Minimum } \\
\text { latency }\end{array}$ & $\begin{array}{l}\text { Maximum } \\
\text { latency }\end{array}$ & \multicolumn{1}{c}{$\begin{array}{l}95 \% \\
\text { latency }\end{array}$} \\
\hline 1) only consumer and provider & $14.4 \mathrm{~ms}$ & $4.9 \mathrm{~ms}$ & $5 \mathrm{~ms}$ & $24 \mathrm{~ms}$ & $22 \mathrm{~ms}$ \\
\hline $\begin{array}{c}\text { 2) consumer, provider and } \\
\text { ten additional medical devices }\end{array}$ & $14.6 \mathrm{~ms}$ & $5.0 \mathrm{~ms}$ & $5 \mathrm{~ms}$ & $24 \mathrm{~ms}$ & $22 \mathrm{~ms}$ \\
\hline $\begin{array}{c}\text { 3) consumer, provider, ten } \\
\text { medical devices and } \\
\text { six simulated devices }\end{array}$ & $19.8 \mathrm{~ms}$ & $8.7 \mathrm{~ms}$ & $5 \mathrm{~ms}$ & $57 \mathrm{~ms}$ & $36 \mathrm{~ms}$ \\
\hline $\begin{array}{l}\text { 4) consumer, provider, ten } \\
\text { medical devices and } \\
31 \text { simulated devices }\end{array}$ & $106.2 \mathrm{~ms}$ & $129.6 \mathrm{~ms}$ & $5 \mathrm{~ms}$ & $1,188 \mathrm{~ms}$ & $362 \mathrm{~ms}$ \\
\hline \hline $\begin{array}{l}\text { Reference measurement } \\
\text { without the OR.NETwork }\end{array}$ & $4.3 \mathrm{~ms}$ & $0.9 \mathrm{~ms}$ & $3 \mathrm{~ms}$ & $8 \mathrm{~ms}$ & $6 \mathrm{~ms}$ \\
\hline
\end{tabular}

Table 1: Results of the latency tests with different test scenarios.

\section{Discussion}

The results show that the required latency of less than $75 \mathrm{~ms}$ can be achieved easily with a tracking camera integrated to the OR.NETwork, as long as the network is not at its capacity limit like in scenario four. Comparing the latencies measured in scenario one to the reference measurement, there is a time loss between $2 \mathrm{~ms}$ and $16 \mathrm{~ms}$. This is mainly due to the limited update rate of $60 \mathrm{~Hz}$ (equaling one new frame every $16.7 \mathrm{~ms}$ ) with which the tracking data is transmitted over the OR.NETwork. Comparing scenario one to scenario two reveals that just the traffic generated by the discovery messages of the additional devices inside the network has no significant effect on the latency. Even providers sending out data periodically and simultaneous user interaction being performed with other medical devices, as measured in scenario three, do not interfere with the navigational task.

Only for robotic applications the increased maximum latency and standard deviation of scenario three could lead to disturbances of the control algorithms. A real time add-on to the OR.NET-SDCstandard with a second parallel network in place, as proposed in (Vossel 2017) and (Pfeiffer 2018), could eliminate this issue. Another solution would be to use the same physical network but different channels for real time and non real time traffic as presented in (Cucinotta 2009). In the OR.NET context, those channels could be administered by a dedicated OR.NET switch. The switch would be aware of connected real time devices and in predefined time intervals (e.g. every millisecond) a time slot of for example $100 \mu$ s could be reserved for real time traffic. In the remaining time, the normal traffic of the service oriented OR.NETwork can be handled. This way only one physical network is required and backwards compatibility is given to OR.NET ready devices that do not comply with a future real time add-on of the standard. The evaluation of this approach is one objective of our ongoing work.

The proposed real time add-on could then also solve disturbances of the navigational task when the OR.NETwork is at its capacity limit, as tested in scenario four. On the other hand, pushing the network to its limit is probably only performed when transferring large amounts of data such as $3 \mathrm{D}$ images or $4 \mathrm{k}$ video streaming, which is unlikely to happen during the navigational procedure.

The tests presented in this paper are a first estimation and have some minor drawbacks. When comparing the latency results to literature, the time required to show the position of the instruments on 
the display, including the display update rate, is not included. This time, which highly depends on the hardware of the navigation system and the connected monitor, must be added to the results shown in Table 1.

Further tests should be carried out that do not only measure the bare latency of the tracking device and the OR.NETwork but also give a navigational task to a group of users. These usability tests are further aspects of our ongoing work. In parallel, related safety concepts and strategies are iteratively evaluated with notified bodies in Germany and the Food and Drug Administration in the US ( $\operatorname{Jan} ß 2018$; Blaar 2016; Zeißig 2016) in the scope of the OR.NET initiative (www.ornet.org). The work presented in this paper contributes to these efforts.

\section{References}

Besting A, Bürger S, Kasparick M, Strathen B, Portheine F, Software design and implementation concepts for an interoperable medical communication framework, Biomedical Engineering / Biomedizinische Technik, 63(1), pp.49-56, 2018.

Blaar M, Janß A, Dell'Anna J, Höllig A, Radermacher K, Clusmann H, Bottlenecks and needs in human-human and human-machine interaction - a view from and into the neurosurgical OR, Biomedical Engineering / Biomedizinische Technik, 61(2), 2016.

Chen JYC, Haas EC, Barnes MJ, Human performance issues and user interface design for teleoperated robots, IEEE Transactions on Systems, Man and Cybernetics Part C: Applications and Reviews, 37(6), pp.1231-1245, 2007.

Cucinotta T, Mancina A, Anastasi GF, Lipari G, Mangeruca L, Checcozzo R, Rusinà F, A real-time service-oriented architecture for industrial automation, IEEE Transactions on Industrial Informatics, 5(3), pp.267-277, 2009.

Dell'Anna J, Janß A, Clusmann H, Radermacher K, A Configurable Footswitch Unit for the Open Networked Neurosurgical OR - Development, Evaluation and Future Perspectives, I-Com, 15(3), pp.227-247, 2016.

Fuente M de la, Belei P, Ohnsorge JA, Skwara A, Radermacher K, Zero-Dose C-arm navigation: an efficient approach based on virtual X-ray targeting, In Langlotz, F., Davies, B., Grützner, P. A., eds. Proceedings of CARS 2007. Berlin, Germany, pp. 249-250. 2007.

Golatowski F, Janß A, Leucker M, Neumuth T, OR.NET - secure dynamic networks in the operating room and clinic, Biomedical Engineering / Biomedizinische Technik, 63(1), pp.1-3, 2018.

Ibach B, Konzeption und Entwicklung einer serviceorientierten Integrationsarchitektur für die Vernetzung von Medizinprodukten im Operationssaal, Shaker, Aachen, 2011.

Ibach B, Benzko J, Janß A, Radermacher K, Sharing tracking data between different systems using an Open Surgical Communication Bus, In Dössel, O., Schlegel, W. C., eds. IFMBE Proceedings. Munich: Springer-Verlag, pp. 21-23. 2009.

Ibach B, Zimolong A, Bullita C, Radermacher K, Concept of a service-oriented integration architecture for the orthopaedic operating theatre, International Journal of Computer Assisted Radiology and Surgery, 3(Supplement 1), pp.S446-S447, 2008.

Janß A, Ibach B, Lauer W, Radermacher K, Performance evaluation of a multi-purpose input device for computer-assisted surgery, In IFMBE Proceedings. pp. 183-185. 2009.

Janß A, Thorn J, Schmitz M, Mildner A, Dell'Anna-Pudlik J, Leucker M, Radermacher K, Extended device profiles and testing procedures for the approval process of integrated medical devices using the IEEE 11073 communication standard, Biomedical Engineering / Biomedizinische Technik, 63(1), pp.95-103, 2018.

Kasparick M, Schlichting S, Golatowski F, Timmermann D, New IEEE 11073 standards for interoperable, networked point-of-care Medical Devices, In 2015 37th Annual International Conference of the IEEE Engineering in Medicine and Biology Society (EMBC). pp. 1721-1724. 
2015.

Ladenburger A, Nebelung S, Buschmann C, Strake M, Ohnsorge JA, Radermacher K, Fuente M de la, Zero-Dose C-Arm-Navigation (ZDCAN) as a Tool for Education: Radiation Dose Reduction in Lumbar Interventional Techniques, In Orthopaedic Proceedings. p. 46. 2012.

Luck JP, McDermott PL, Allender L, Russell DC, An Investigation of Real World Control of Robotic Assets under Communication Latency, Proceeding of the 1st ACM SIGCHI/SIGART conference on Human-robot interaction - HRI '06, p.202, 2006.

MacKenzie IS, Ware C, Lag As a Determinant of Human Performance in Interactive Systems, In Proceedings of the INTERACT ' 93 and CHI '93 Conference on Human Factors in Computing Systems. CHI '93. New York, NY, USA: ACM, pp. 488-493. 1993.

Mildner A, Janß A, Dell'Anna-Pudlik J, Merz P, Leucker M, Radermacher K, Development of Deviceand Service-Profiles for a Safe and Secure Interconnection of Medical Devices in the Integrated Open OR, In Seehusen, F., Felderer, M., Großmann, J., Wendland, M.-F., eds. Risk Assessment and Risk-Driven Testing. New York: Springer International Publishing, pp. 65-74. 2015.

Pfeiffer JH, Kasparick M, Strathen B, Dietz C, Dingler ME, Lueth TC, Timmermann D, Radermacher K, Golatowski F, OR.NET RT: how service-oriented medical device architecture meets real-time communication, Biomed Tech (Berl), 63(1), pp.81-93, 2018.

Vitting A, Janß A, Strathen B, Strake M, Radermacher K, Further Development and Evaluation of a Universal Foot Switch for Diverse Medical Disciplines within the Framework of an Open Integration Concept for the Operation Theatre of the Future, In Duffy, V., Lightner, N., eds. Advances in Human Factors and Ergonomics in Healthcare and Medical Devices. Cham: Springer International Publishing, pp. 438-449. 2018.

Vossel M, Strathen B, Kasparick M, Müller M, Radermacher K, Fuente M de la, Janß A, Integration of Robotic Applications in Open and Safe Medical Device IT Networks Using IEEE 11073 SDC, In Radermacher, K., Baena, F. R. Y., eds. CAOS 2017. 17th Annual Meeting of the International Society for Computer Assisted Orthopaedic Surgery. EPiC Series in Health Sciences. EasyChair, pp. 254-257. 2017.

Zeißig E-M, Janß A, Dell'Anna J, Ziefle M, Radermacher K, Development and experimental evaluation of an alarm concept for an integrated surgical workstation, Biomedical Engineering / Biomedizinische Technik, 61(2), 2016.

\section{Acknowledgements}

This research work has been funded within the MoVE project (MoVE - Modular Validation Environment for Medical Device Networks, German Ministry for Education and Research (BMBF); grant no.: 03VNE1036D). 